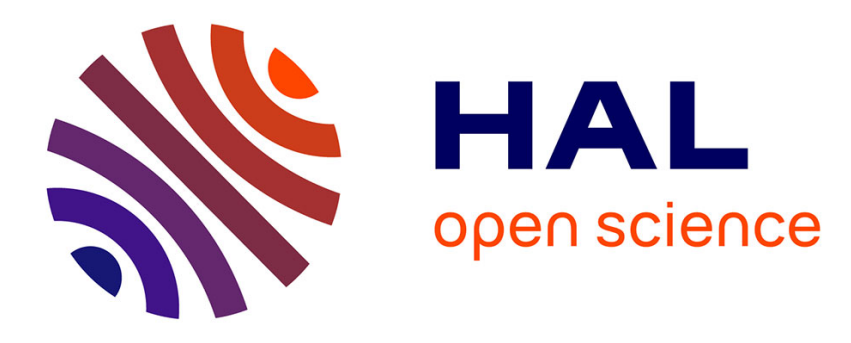

\title{
A survey on energy efficient techniques in wireless sensor networks
}

Ridha Soua, Pascale Minet

\section{To cite this version:}

Ridha Soua, Pascale Minet. A survey on energy efficient techniques in wireless sensor networks. WNNC 2011 - 4th Joint IFIP Wireless and Mobile Networking Conference, Oct 2011, Toulouse, France. pp.1 - 9, 10.1109/WMNC.2011.6097244 . hal-00863351

\section{HAL Id: hal-00863351 https://hal.science/hal-00863351}

Submitted on 20 Sep 2013

HAL is a multi-disciplinary open access archive for the deposit and dissemination of scientific research documents, whether they are published or not. The documents may come from teaching and research institutions in France or abroad, or from public or private research centers.
L'archive ouverte pluridisciplinaire $\mathbf{H A L}$, est destinée au dépôt et à la diffusion de documents scientifiques de niveau recherche, publiés ou non, émanant des établissements d'enseignement et de recherche français ou étrangers, des laboratoires publics ou privés. 


\title{
A Survey on Energy Efficient Techniques in Wireless Sensor Networks
}

\author{
Ridha Soua and Pascale Minet \\ INRIA \\ Rocquencourt \\ 78153 Le Chesnay cedex, France \\ Email: ridha.soua@inria.fr, pascale.minet@inria.fr
}

\begin{abstract}
The myriad of potential applications supported by wireless sensor networks (WSNs) has generated much interest from the research community. Various applications range from small size low industrial monitoring to large scale energy constrained environmental monitoring. In all cases, an operational network is required to fulfill the application missions. In addition, energy consumption of nodes is a great challenge in order to maximize network lifetime. Unlike other networks, it can be hazardous, very expensive or even impossible to charge or replace exhausted batteries due to the hostile nature of environment.

Researchers are invited to design energy efficient protocols while achieving the desired network operations. This paper focuses on different techniques to reduce the consumption of the limited energy budget of sensor nodes. After having identified the reasons of energy waste in WSNs, we classify energy efficient techniques into five classes, namely data reduction, control reduction, energy efficient routing, duty cycling and topology control. We then detail each of them, presenting subdivisions and giving many examples. We conclude by a recapitulative table.
\end{abstract}

\section{INTRODUCTION}

Wireless sensor networks (WSNs) consist of a possibly large amount of wireless networked sensors required to operate in a possibly hostile environment for a maximum duration without human intervention. Typically, a sensor node is a miniature device that includes four main components: a sensing unit for data acquisition, a microcontroller for local data processing and some memory operations, a communication unit to allow the transmission/reception of data to/from other connected devices and finally a power source which is usually a small battery. WSNs support a wide range of applications such as target tracking, environmental monitoring, system control, health monitoring or exploration in hostile environment. For data gathering applications, which represent the main use of WSN applications, the goal is to detect any event occurring in the area of interest and to report it to the sink. [1], [2] are the earliest papers proving that if the communication range is at least twice the sensing range, a full coverage implies connectivity among active nodes inside the area of interest.

Application scenarios for WSNs often involve batterypowered nodes being active for a long period, without external human control after initial deployment. In the absence of energy efficient techniques, a node would drain its battery within a couple of days. This need has led researchers to design protocols able to minimize energy consumption. In [3], authors present a taxonomy of energy conservation schemes. Their very interesting classification, however, does not include energy efficient routing, protocol overhead reduction, data aggregation and cross-layering mechanisms. In this survey, we cope with this lack by providing a new classification integrating more techniques.

The reminder of this paper is organized as follows. Section II defines network lifetime, the crucial concept behind any energy efficient technique. The aim of Section III is to understand the different sources of energy waste in WSN and to categorize energy efficient techniques according to the solved problems. Sections IV to VIII describe these techniques in details. We conclude in Section IX with a recapitulative table.

\section{NETWORK LIFETIME DEFINITION}

The most challenging concern in WSN design is how to save node energy while maintaining the desirable network behavior. Any WSN can only fulfill its mission as long as it is considered alive, but not after that. As a consequence, the goal of any energy efficient technique is to maximize network lifetime. This latter depends drastically on the lifetime of any single node. However, in the literature, there is no consensus for the definition of network lifetime. The majority of authors use a definition suitable for the context of their work. This situation has driven toward a plethora of coexisting definitions. Based on the previous works on WSNs [4], [5], we give an overview of the most common definitions.

1) Network lifetime based on the number of alive nodes The definition found most frequently in the literature is the time during which all sensors are alive (also called $n$ out of $n$ in [5], where $n$ is the total number of sensors). The sink nodes are excluded from the set of nodes to reflect the assumption that sink nodes are more sophisticated and powerful devices. This lifetime is easy to compute since it does not take into account the topology changes. However, in dense networks where redundancy is present, this metric does not represent actually the lifetime evaluation. Therefore, the only case in which this metric can be reasonably used is if all nodes are of equal of importance and critical to network application. 
A variant defines the network lifetime as the time until the fraction of alive nodes falls below a predefined threshold $\beta$ [6]. While this definition takes redundancy into account unlike the former, it does not accurately describe the correct running of data gathering applications where the failure of at most $\beta \%$ of sensors near the sink can prevent the sink to receive collected data. In the context of clustering [7], [8], authors define the network lifetime as the time to failure of the first cluster head. However, in most works, researchers change cluster head dynamically to balance energy consumption.

2) Network lifetime based on coverage

Coverage reflects how well the network can detect an event in the monitored area. Therefore some works define the lifetime as the time during which the area of interest is covered by sensor nodes. However, even an $100 \%$ coverage is not sufficient because it does not ensure that collected data are delivered to the sink.

3) Network lifetime based on connectivity

This definition is based on the ability of the network to transmit data to a sink. This definition is similar to what has been proposed in context of ad hoc networks. In [9] authors define the lifetime as the minimum time when either the percentage of alive nodes or the size of the largest connected component of the network drops below a specific threshold.

4) Network lifetime based on application requirements Some authors consider that network is alive as long as application functionalities are ensured. Kumar et al. [10] state "we define the lifetime of a WSN to be the time period during which the network continually satisfies the application requirements". Tian and Georganas [6] suggest another definition: It is the time until "the network no longer provides an acceptable event detection ratio." However, if no connectivity is guaranteed to report the event, this definition becomes irrelevant.

As a conclusion, network lifetime must take into account connectivity and coverage if needed by the application supported by WSN. Knowledge of the application requirements will enable WSN designers to refine the definition of network lifetime, leading to an evaluation more realistic and more pertinent for the application users.

\section{TAXONOMY OF ENERGY EFFICIENT TECHNIQUES}

We detail in this section the reasons of potential energy waste in a WSN. We then propose a taxonomy of existing energy efficient solutions, keeping in mind the resource constraint nature of sensors.

\section{A. Reasons of energy waste}

In WSNs, sensors dissipate energy while sensing, processing, transmitting or receiving data to fulfill the mission required by the application. The sensing subsystem is devoted to data acquisition. It is obvious that minimizing data extracted from transducer will save energy of very constrained sensors. Redundancy inherent to WSNs will produce huge similar reporting that the network is in charge of routing to the sink. Experimental results confirm that communication subsystem is a greedy source of energy dissipation.

With regard to communication, there is also a great amount of energy wasted in states that are useless from the application point of view, such as [4]:

- Collision: when a node receives more than one packet at the same time, these packets collide. All packets that cause the collision have to be discarded and the retransmission of these packets is required.

- Overhearing: when a sender transmits a packet, all nodes in its transmission range receive this packet even if they are not the intended destination. Thus, energy is wasted when a node receives packets that are destined to other nodes.

- Control packet overhead: a minimal number of control packets should be used to enable data transmissions.

- Idle listening: is one of the major sources of energy dissipation. It happens when a node is listening to an idle channel in order to receive possible traffic.

- Interference: each node located between transmission range and interference range receives a packet but cannot decode it.

As network lifetime has become the key characteristic for evaluating WSN, a panoply of techniques aimed at minimizing energy consumption and improving network lifetime, are proposed. We now give a taxonomy of these techniques.

\section{B. Classification of energy efficient techniques}

We can identify five main classes of energy efficient techniques, namely, data reduction, protocol overhead reduction, energy efficient routing, duty cycling and topology control.

1) Data reduction: focuses on reducing the amount of data produced, processed and transmitted. For instance, data compression and data aggregation are examples of such techniques.

2) Protocol overhead reduction: the aim of this technique is to increase protocol efficiency by reducing the overhead. Different techniques exist. Transmission periods of messages are adapted depending on the stability of the network, or on the distance to the source of the transmitted information. More generally, a cross-layering approach will enable an optimization of the communication protocols taking into account the application requirements. Another technique, optimized flooding can significantly contribute to reduce the overhead.

4 Energy efficient routing: routing protocols should be designed with the target of maximizing network lifetime by minimizing the energy consumed by the end-to-end transmission and avoiding nodes with low residual energy. Some protocols are opportunistic, taking advantage of node mobility or the broadcast nature of wireless communications to reduce the energy consumed by a transmission to the sink. Others use geographical coordinates of nodes to build a route toward the destination. 
TABLE I: Impact of energy efficient techniques on sources of energy waste.

\begin{tabular}{|c|c|c|c|c|c|c|c|}
\hline $\begin{array}{ll}\text { Techniques } & \text { Reasons of energy waste } \\
\text { The }\end{array}$ & $\begin{array}{l}\text { Sensing - } \\
\text { processing }\end{array}$ & Communication & Collision & Overhearing & $\begin{array}{l}\text { Control- } \\
\text { packets }\end{array}$ & $\begin{array}{l}\text { Idle } \quad- \\
\text { listening }\end{array}$ & Interference \\
\hline Data reduction & $M$ & $M$ & $S$ & $S$ & - & - & $S$ \\
\hline Protocol overhead reduction & - & $M$ & $S$ & $S$ & $M$ & - & $S$ \\
\hline Energy efficient routing & - & $M$ & $S$ & $M$ & $S$ & - & $M$ \\
\hline Duty cycling & $M$ & $M$ & $M$ & $M$ & $S$ & $M$ & $M$ \\
\hline Topology control & - & $M$ & $M$ & $M$ & - & - & $M$ \\
\hline
\end{tabular}

Others build a hierarchy of nodes to simplify routing and reduce its overhead. Finally, data centric protocols send data only to interested nodes in order to spare useless transmissions.

3 Duty cycling: duty cycling means the fraction of time nodes are active during their lifetime. Nodes sleep/active schedules should be coordinated and accommodated to specific applications requirements. These techniques can be further subdivided. High granularity techniques focus on selecting active nodes among all sensors deployed in the network. Low granularity techniques deal with switching off (respectively on) the radio of active nodes when no communication is required (respectively when a communication involving this node may occur). They are highly related to the medium access protocol.

4 Topology control: it focuses on reducing energy consumption by adjusting transmission power while maintaining network connectivity. A new reduced topology is created based on local information.

Table I shows how each energy efficient technique class tackles sources of energy waste. The 'M' symbol means a main impact, whereas a ' $S$ ' symbol means a secondary impact. We now detail these different classes.

\section{DATA REDUCTION}

Data reduction techniques proposed in the literature can be classified into three categories according to the data handling step: production, processing and finally communication step. All categories are detailed in separate subsections.

\section{A. Production step}

In many cases, data generated by active nodes rarely change during network lifetime. This has spurred researchers to exploit temporal correlation of sensed data: prediction techniques have emerged. In addition, investigated environments are often dynamic and can witness changes in different areas. The challenge is to represent an accurate picture of the true state of the world while making an efficient use of resources. This has given birth to different techniques.

1) Sampling based techniques: By reducing the data sampled by sensor nodes, we decrease not only the radio subsystem energy consumption but also the communication cost. A lot of work in sampling techniques has been done. We focus on adaptive sampling techniques. The interested reader can refer to [3] for a comprehensive survey about hierarchical and model based sampling.
Adaptive sampling techniques exploit the spatio-temporal correlation between samples to make data collection rate dynamic. This can drastically reduce the amount of data extracted from transducer. Three different approaches can be found in adaptive sampling:

- God view: a central node knows data characteristics and sends the appropriate sampling rate to sensor nodes. Specifically, the sink must have a global knowledge about the network and the environment [14].

- Full autonomous nodes: each node adjusts its sampling rate based on the input data characteristics [15].

- Partial autonomous nodes: remote sources are allowed to modify the sampling interval independently within a specified range. If the desired modification of the sampling interval is more than the allowed range, a new sampling rate is requested from the sink [16].

2) Prediction based techniques: Given the past history of readings and based on the observation that sensors are capable of local computation, a sink can usually predict the set of readings and so the sensing device can be turned off. Data prediction techniques are based on a data model: Queries are answered locally using a model instead of transmitting the sensed data. Hence, sensors do not need to transmit the sensed data as long as they are within a certain threshold or error bound. Prediction based techniques can be broadly classified in the following two categories: centralized and clustering based. An example of centralized prediction technique is given in [11]. Goel and al show that data prediction can be visualized as a watching of a sensor movie and hence MPEG concepts can be applied. Sensor nodes send their readings to the sink. This latter computes the model based on the correlation between macro blocks and sends it back to sensors. Future sensor readings will be compared to this model and only readings out of bound will be transmitted to the sink. In the second class that benefits from spatial correlation, authors of ASAP [12] propose that nodes with similar data readings are associated with the same cluster. The cluster head as well as the sink maintain a prediction model. The cluster head compares the sensed data with the model prediction. Only out of bound sensed data are transmitted to the sink. The buddy protocol [13] establishes a buddy relationship between a node and its neighbors to exploit the spatio-temporal correlation of sensed data. This gives birth to a number of buddy groups with a buddy head in charge of monitoring and processing queries. 


\section{B. Processing and Communication step}

Different operations on collected data have been introduced during the processing step to handle the scarcity of energy resources in a WSN. We focus on two main techniques : data compression and data aggregation.

1) Data compression: Since data communication is the most exhausting task that a sensor undertakes, data compression reduces the number of bits to be transmitted and relayed by battery powered devices. Therefore, the network lifetime can be significantly extended. There are multiple techniques to compress data [17]. The most relevant techniques tailored to WSN can be classified as:

- Coding by ordering: in this technique, data from multiple sensors are combined at a compression node. Some data of specific nodes are dropped. However, the dropped data can be computed from the coding order of the included data.

- Pipelined in network compression: extracted data are stored in a compression node buffer for some time interval. The compression node exploits this period to combine data packets into a single packet. Redundancy will be removed to reduce the amount of data that must be transmitted across the network.

- Distributed Compression: it consists of compressing sensor data from individual nodes while requiring minimal (or no) inter-sensor communication. For instance, two sources of correlated information send encoded data to a third node in charge of reconstructing the two original data.

2) Data aggregation: As sensors tend to be more and more miniature, data storage memory component is expected to be smaller and smaller. Therefore, many studies have been conducted to eliminate redundancy and reduce data towards the sink. Specifically, aggregation techniques deal with distributed processing of data and coordination among nodes to achieve better performances. Existing solutions can be classified into three major categories:

- Cluster based structure : nodes are organized in clusters and the cluster heads are responsible of data aggregation. Then cluster heads communicate directly with the sink. LEACH protocol was the first work to propose this structure [18], [19]. PEGASIS enhances LEACH by organizing all nodes in a chain and letting nodes to alternate the head of the chain. Hierarchical-PEGASIS [20] is an extension of PEGASIS.

- Tree based structure : in [21], [22] authors propose DCTC, where each sensor node knows the distance to the event detected. The nearest node of the center of the event is chosen as the root of the aggregation tree. In [23], authors propose an aggregation tree construction based on a simple min-cost perfect matching. Traditional multicast algorithms like SMT (Steiner Minimum Tree) and MST (Multiple Shared Tree) are a source of inspiration for aggregation protocols in WSN [24], [25].

- Structure-less protocol : authors of [26] propose a novel technique without incurring the overhead of a structurebased approach. It uses anycast to forward packet to onehop neighbor that aggregates data packets. This approach is suitable for dynamic event scenarios. Fan et al. [27] propose ToD, a scalable technique that takes benefits from the absence of explicit structure to reduce overhead.

\section{PRotocol overhead REDUCTION}

An important energy waste occurs as a result of protocol overhead. In this section, we discuss the outlines of reducing protocol overhead to save the scarce energy resource and hence extend network lifetime. These techniques can be subdivided into 1) adaptive transmission period depending on WSN stability or distance to the information source, 2) cross-layering with the upper and lower layers to optimize network resources while meeting application requirements and 3) optimized flooding to avoid unnecessary retransmissions.

\section{A. Adaptive transmission period}

Communication protocols often resort to periodic message exchanges. These periodic control messages are sources of overhead in WSN. Reducing the period saves energy and bandwidth but increases protocol latency to changes. The determination of the best period value must take into account this trade-off. Moreover, since the environment of the WSN is dynamic, the period should be adapted to the environment and to the frequency of changes in this environment. Hence, the idea of an adaptive transmission period, depending on the observed changes. Furthermore, some information has an importance degree that decreases when the distance to the information source increases (e.g. car accident on a motorway).

1) Adaptivity to WSN changes: Neighborhood discovery and computation of energy efficient routes, to name a few, are examples of communication protocols where control messages are periodically exchanged. In addition, as communication links can easily be broken due to mobility or node depletion, this ends up with creating more control packets. In [28], authors suggest to adapt the message period to network stability. For instance, two periods HelloMin and HelloMax are used for neighborhood discovery. HelloMax represents the period of sending Hellos in a stable network. This is the maximum and default value that the network tends to reach. Moreover, HelloMin represents the minimum time interval elapsed since the last transmission of a Hello by a node detecting a topology change.

A more sophisticated approach, called Trickle algorithm [29], achieves energy saving in disseminating information after a change. The basic idea behind is to allow two nodes to determine very quickly if they have the same version of data and otherwise to synchronize. If the two nodes are synchronized, there is no more communication. When new information appears, the traffic is resumed.

2) Adaptivity to the distance to the information source: The basic idea is the Fish Eye concept [30] where the period of transmission of an information increases with the distance. Typically, in a routing protocol, information is refreshed every 
period for nodes up to 3-hop from the source, every two periods for nodes from 4-hop to 6-hop, and every four periods for other nodes.

\section{B. Cross layering optimization}

WSN requirements include reliability, responsiveness, power efficiency and scalability. To meet these requirements with resource constrained sensors, a panoply of cross layering approaches has been proposed [31].

- Top-down approach: higher layers dictate parameters and strategies to the lower layer. For example, application layer dictates the MAC parameter while the MAC layer selects the optimal PHY layer modulation scheme.

- Bottom up approach: lower layers do abstraction of losses and bandwidth variations for higher layers. This cross layer solution is not suitable for multimedia applications.

- Application-centric approach: this approach alternates between bottom-up (starting from the physical layer) and top-down manner to optimize the lower layers parameters.

- MAC-centric approach: MAC layer decides the QoS (quality of service) required level and which application flows should be transmitted according to application layer requirements.

- Integrated approach: strategies are determined jointly. However, finding the optimal composite strategy is complex. For multimedia applications, the quality of the multimedia content viewed by users is an indicator of the strategy performance level.

Less radical cross-layering approaches just use information provided by the upper layers and the lower ones to optimize network resources use while meeting the application requirements. For instance, in data gathering applications, the routing protocol maintains only useful routes: routes toward the sink. Furthermore, the QoS perceived by the user will be improved, if the routing protocol uses only links with good quality, this quality being known at the MAC level.

\section{Optimized flooding}

Flooding is a widely used technique in WSN for location discovery, route establishments, querying, etc. However, given the restrictions on energy and bandwidth in WSN, flooding is a very expensive operation for battery powered sensors. In this section, we will discuss techniques whose aim is to limit the number of transmissions generated each time some information must be disseminated in the whole network. We distinguish:

- Multipoint relaying based mechanism: this technique is introduced in the OLSR routing protocol. Only a small set of neighbors of the sending node has to retransmit packets. These nodes are called MultiPoint Relays (MPRs). Indeed, the multipoint relay set of a node is the minimum set of one-hop neighbors covering all its two-hop nodes. A node $N$ forwards a received broadcast message if and only if this message has a non-null timeto-live and has been received for the first time from a node having selected $N$ as multipoint relay.

- Connected dominating sets (CDs) based mechanism: CDs have been used to optimize flooding in MANET. Each node checks if it belongs to $\mathrm{CD}$ or not. If so, it retransmits the broadcast message after having received it. It was proven that finding a minimum connected dominating set is NP-hard for most graphs [32]. Distributed heuristics exist such as [33], [34], [35] where a connected dominating set is built initially and then pruned by removing redundant nodes. Others use the spanning tree of a leader node to assign a rank to each node, such as [36], [37].

- Neighbor negotiation based mechanism: unlike the two previous techniques, the aim is not here to disseminate data throughout the network but to provide it only to interested nodes. For this purpose, neighbors exchange descriptors of received data. Any interested node (a node that wants to receive the data and does not have it) asks for it by sending a query. For instance, in SPIN (Sensor Protocols for Information via Negotiation) [38], any data is described by a descriptor named meta-data which is unique and shorter than the actual data. However, SPIN data forwarding cannot guarantee the delivery of data. This is due to intermediate nodes which can be not interested in the data [20].

\section{ENERGY EFFICIENT ROUTING PROTOCOLS}

The energy constraints of sensor nodes raise challenging issues on the design of routing protocols for WSNs. Proposed protocols aim at load balancing, minimizing the energy consumed by the end-to-end transmission of a packet and avoiding nodes with low residual of energy. In this section, we give a classification rather than an exhaustive list of energy efficient routing protocols. Our classification of energy efficient routing protocols generalizes the one given in [20]: data centric protocols, hierarchical protocols, geographical and opportunistic protocols. Each category will be discussed in details in next subsections.

\section{A. Data centric protocols}

These protocols target energy saving by querying sensors based on their data attributes or interest. They make the assumptions that data delivery is described by a query driven model. Nodes route any data packet by looking at its content. Mainly, two approaches were proposed for interest dissemination. The first is SPIN [38] where any node advertises the availability of data and waits for requests from interested nodes. The second is Directed Diffusion (DD) [20] in which sinks broadcast an interest message to sensors, only interested nodes reply with a gradient message. Hence, both interest and gradients establish paths between sink and interested sensors. Many other proposals have being made such as rumor routing, gradient based routing, COUGAR, CADR. See [20] for a comprehensive summary. 


\section{B. Hierarchical protocols}

Recently, clustering protocols have been developed in order to improve scalability and reduce the network traffic towards the sink. Cluster based protocols have shown lower energy consumption than flat networks despite the overhead introduced by cluster construction and maintenance. One of the pioneering hierarchical routing protocol is LEACH [20]. In this protocol, sensors organize themselves in local clusters with one node acting as a cluster head. To balance energy consumption, a randomized rotation of cluster head is used.

PEGASIS is another example of hierarchical protocol [20]. It enhances LEACH by organizing all nodes in a chain and letting nodes to alternate the head of the chain. TEEN is both data centric and hierarchical. It builds clusters of different levels until reaching the sink. The data centric aspect is outlined by using two thresholds for sensed attributes: Hard threshold and soft threshold. The former will trigger the sensor node to transmit to its cluster head. Another transmission is only permitted when the attribute value becomes higher than the soft threshold. This mechanism can drastically reduce the number of transmission and thus energy consumption. Since TEEN is not adaptive to periodic sensor data reporting, an extension called APTEEN [20] has been proposed.

\section{Geographical protocols}

Non geographical routing protocols suffer from scalability and efficiency restrictions because they depend on flooding for route discovery and updates. Geographical protocols take advantage of nodes location information to compute routes. In [20], authors propose an energy aware protocol called GEAR consisting of two phases. In the first phase, the message is forwarded to the target region. In the second phase, the message is forwarded to the destination within the region.

The basic idea behind GEAR is to enhance DD by sending the interests only to a certain region rather than the whole network. GAF [20] ensures energy efficiency by building virtual grids based on location information of nodes. Only a single node needs to be turned on in each cell, other nodes are kept in sleeping state. SPEED [18] ensures load balancing among multiple routes with its non deterministic forwarding module.

\section{Opportunistic protocols}

The crucial idea of opportunistic routing is to exploit 1) the broadcast nature and space diversity provided by the wireless medium or 2) node mobility. We distinguish two subclasses of opportunistic routing:

1) Medium broadcast nature and space diversity based protocols: These techniques maintain multiple forwarding candidates and judiciously decide which sets of nodes are good and prioritized to form the forwarding candidate set. In [39], authors highlight how these protocols achieve better energy efficiency.
2) Mobility based protocols: By introducing mobility in WSN, network lifetime can be extended. Indeed, mobile nodes can move to isolated parts of the network and hence connectivity is again reached. Several works merging routing and mobility have demonstrated that this class of routing protocol exhibits smaller energy consumption when compared to classical techniques.

- Mobile sink based protocols: the authors of [40] propose a framework where mobility of the sink and routing are joint. Their proposed routing strategy offers $500 \%$ improvement of network lifetime by using combination of sink trajectory and short paths. In [41], [42], a learningbased approach is proposed to efficiently and reliably route data to a mobile sink. Sensors in the vicinity of the sink learn its movement pattern over time and statistically characterize it as a probability distribution function. In [43], authors demonstrate that maximum lifetime can be achieved by solving optimally two joint problems: a scheduling problem that determines the sojourn times of the sink at different locations, and a routing problem in order to deliver the collected data to the sink in an energy efficient way.

- Mobile relay based protocols: these techniques have been introduced in the context of opportunistic networks [44] where the existence of an end-to-end routing path is not usually ensured. Thus, any node can be used as an intermediate hop for forwarding data closer to the destination. In [45], authors assume the existence of mobile entities (called MULES) present in the monitored area. MULEs pick up data from the sensors when in close range, buffer it, and drop off the data to wired access points. Their model integrates a random walk for mobility pattern and incorporates system variables such as number of MULEs, sensors and access points. In [46] data mules accommodate their trajectories for data delivery based on only local information.

\section{DUTY CYCLING}

Duty cycling techniques are also called node activity scheduling techniques. They allow nodes to alternate activity and sleep periods. Indeed, only the sleep state guarantees energy saving since transmitting, receiving and idle listening consumes the scarce and expensive battery power resource. The idea is then to power off the radio subsystem each time it is possible while ensuring an operational network from the application point of view. These techniques can be applied at a high or a low granularity level. Each of them will use different means that will be briefly described.

\section{A. High granularity}

Generally, a large number of sensors is deployed on the monitored area. This high density leads to large redundancy. Therefore, redundant nodes should be switched off to achieve a high level of energy saving while a reduced set of nodes are kept in active mode to meet application requirements. Several works address this challenge. In [47], [48] the selection of 
minimum set of active nodes able to guarantee coverage is based on linear programming techniques. In GAF [20], the monitored area is considered as a virtual grid and divided into small cells. Within each cell, only one node called the leader needs to be active and the other nodes can sleep. However, only connectivity requirements between cells are taken into account. SPAN [51] is a connectivity driven protocol guaranteed by a coordinator eligibility criterion. Coordinators play a vital role by performing multi-hop routing while other sensors can be turned off. In [52], the selection criteria of active nodes are based on both coverage and connectivity requirements. SPAN is enhanced by integrating a Coverage Configuration Protocol (CCP) that can provide different degrees of coverage requested by applications.

Differently of other approaches, authors of [53] divide the network nodes in disjoint sets. Each set should fulfill application requirements. At any time only one set is active while other nodes belonging to other sets can sleep. It has been proven that maximizing the number of disjoint sets is a NP-complete problem. In contrast with the work discussed above, authors of [54] suggest maximizing network lifetime by dividing deployed sensor nodes into non disjoint sets.

\section{B. Low granularity}

This level deals with scheduling activity of nodes which have been selected as active to ensure network functionality. Even these nodes can sleep when they have no message to send or receive. Hence, node activity scheduling should be coordinated with medium access. We distinguish three classes of MAC protocols.

- TDMA-based: time is divided into slots distributed among the nodes. Each slot is used to send or receive data. This technique ensures a collision free medium access to sensor nodes. It is suitable for periodic traffic. TRAMA [55] is the earliest proposed traffic-adaptive TDMAbased protocol. For each time slot, one transmitter within two-hop neighbors is selected based on a distributed algorithm. Time is divided into a random access period to compete for slots and a scheduled access period. FLAMA [56] is derived from TRAMA and dedicated to data gathering applications. FLAMA avoids the periodic information exchange between two-hop neighbors by transmitting upon request only. FlexiTP [57], also proposed in the context of data gathering application, builds a data gathering tree and uses a depth first search of the tree to assign slots. Nodes can claim or remove slots based on the current information in their lookup table. A recent based TDMA protocol called TDMA-ASAP [58], proposed also in the context of data gathering application, integrates a coloring algorithm with the medium access. By allowing a node to steal an unused slot to its brother in the tree, this protocol can be adapted to various traffic conditions.

- Contention-based: S-MAC [59] tries to force neighbor nodes to adopt the same active/sleep schedule. For that purpose, neighbor nodes exchange their schedules using
SYNC messages sent in the first subperiod. The second subperiod is dedicated to data exchange. However, listen and sleep periods of the protocols cannot be varied after node deployment. For this end, T-MAC [60] enhances SMAC by allowing nodes to sleep again if no message has been received for a specified duration. The motivation of D-MAC [61] is to guarantee that all nodes on a multihop path to the sink are awake when the data delivery is in progress. D-MAC schedules the active/sleep period based on its depth on the forwarding tree. To reduce synchronization overhead, asynchronous sleep/wakeup schemes are based on periodic listening. In B-MAC [62], nodes wake up to check the channel for activity and remain active only for a short duration in the absence of traffic.

- Hybrid: protocols of this category switch between TDMA and CSMA to accommodate to variable traffic patterns. The most known is Z-MAC [63]. It runs CSMA in low traffic and switches to TDMA in high traffic conditions. TDMA/CA [64] is a medium access taking advantage of node colors provided by SERENA to offer spatial reuse of the bandwidth and to minimize data delivery time to the sink in case of data gathering.

It appears that graph coloring can be used to improve TDMA efficiency by allowing all nodes/edges with the same color to transmit simultaneously. We distinguish two classes of coloring: node coloring and edge coloring. While the latter assigns time slots per link such that only the transmitter and the receiver are on, the former assigns the slot to the node which is transmitting. Centralized as well as distributed coloring algorithms exist. Some are deterministic, other resort to randomization to color the network. The smaller the number of colors, the better the coloring algorithm. In 2-hop coloring, no two nodes at one or two hops have the same color.

\section{TOPOLOGY CONTROL}

The fundamental idea behind topology control is to build and maintain a reduced topology that will save the small energy budget of sensors while preserving network connectivity and coverage [65]. This can be achieved by reducing the transmission power of sensors. In [66] authors prove that there is an optimal transmission range that minimizes energy dissipation while keeping a connected topology. Since in most applications, devices in WSNs are heterogeneous, we present three topology control algorithms for heterogeneous WSN: Directed LMST (DLMST), Directed RNG (DRNG), and the Residual Energy Aware Dynamic (READ) [67] topology construction algorithm. Both DLMST and DRNG build the reduced topology based on locally collected information. If the original network is strongly connected and symmetric, the reduced topologies computed by these protocols preserve these properties.

On the other side, READ takes benefit from the heterogeneity of nodes where more powerful devices plays a more leading role in the network connectivity to extend network lifetime. 


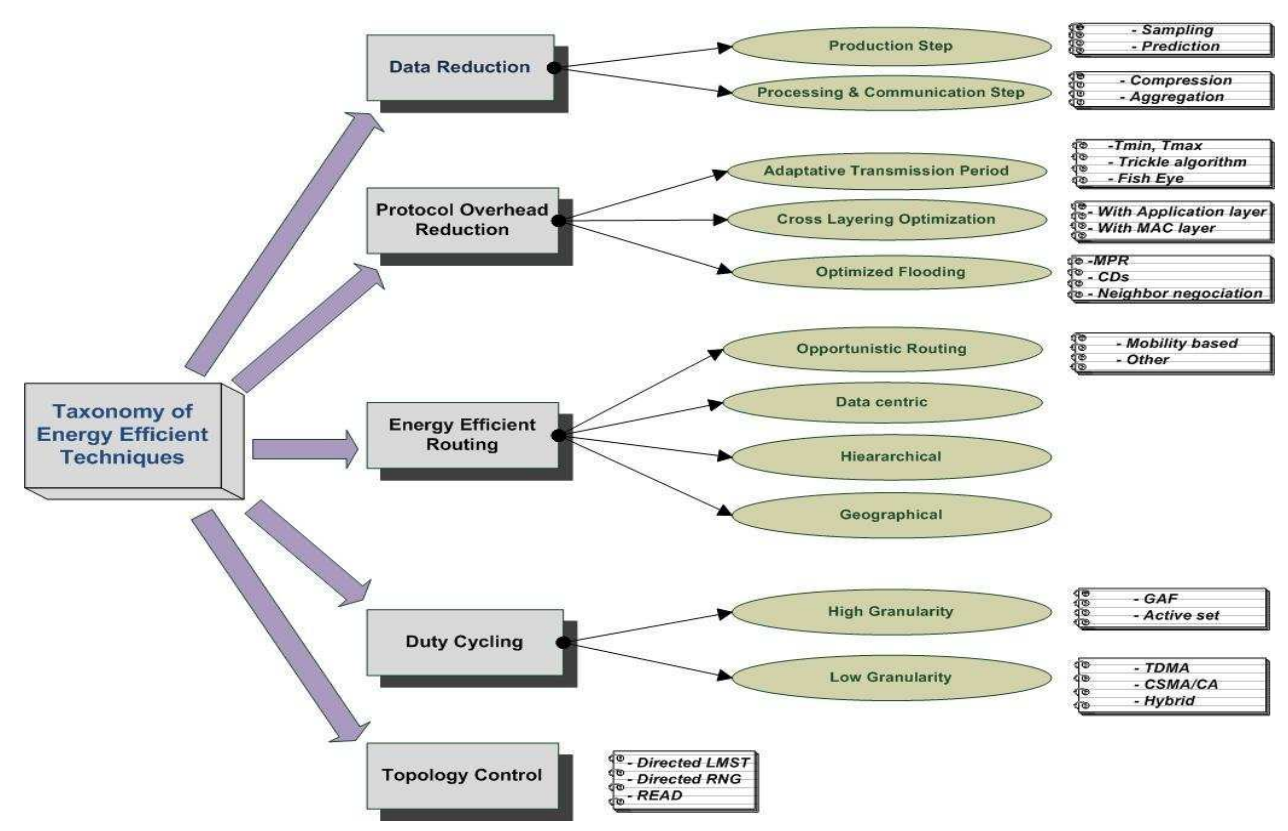

Fig. 1: Taxonomy of energy efficient techniques

Instead of using the euclidian distance between two communicating nodes to define the link cost, READ introduces a weighted cost for each pair of nodes that considers both the energy for sending and receiving data and the current residual energy at each node.

\section{CONCLUSION}

The availability of sensor devices allow a wide variety of applications to emerge. However, the resource constrained nature of sensors raises the problem of energy: how to maximize network lifetime despite a very limited energy budget? In this paper, we have summarized different techniques that tackle the energy efficiency challenge in WSNs and classified them in five main classes as shown in Figure 1 that summarizes this survey. For each class of techniques, we have pointed out which source of energy waste it alleviates.

\section{REFERENCES}

[1] Y. S. Han, W. B. Heinzelman, P. K. Varshney, "Scheduling sleeping nodes in high density cluster-based sensor networks". Journal of Mobile Networks and Applications, Vol.10, December 2005.

[2] X. Wang, G. Xing, Y. Zhang, C. Lu, R. Pless, C. D. Gill, "Integrated coverage and connectivity onfiguration in wireless sensor networks", in Proc. First ACM Conference on Embedded Networked Sensor Systems (SenSys 2003), Los Angeles, November 2003.

[3] G. Anastasi, M. Conti, L. M. Di Franchesco, A. Passarella, "Energy conservation in wireless sensor networks: A survey". Ad Hoc Networks, vol. 7, pp 537-568, 2009.

[4] P. Minet, "Energy efficient routing", in Ad Hoc and Sensor Wireless Networks: Architectures: Algorithms and Protocols. Bentham Science, 2009.

[5] I. Dietrich, F. Dressler, "On the lifetime of Wirless Sensor Networks". ACM Transacions on Sensor Networks, Vol.5, 2009.

[6] D. Tian, N. D. Georganas, "A coverage-preserving node scheduling scheme for large wireless sensor networks", in Proc. the 1st ACM International Workshop on Wireless Sensor Networks and Applications(WSNA), 32-41, 2002.
[7] S. Soro, W. B. Heinzeiman, "Prolonging the lifetime of wireless sensor networks via unequal clustering", in Proc. the 19th IEEE Inetrnational Parallel and distributed Processing Symposium (IPDPS), 2005.

[8] D. M. Blough, P. Santi, "Investigating upper bounds on network lifetime extension for cell-based energy conservation techniques in stationary ad hoc networks", in Proc. the 8th ACM International Conference on Mobile Computing and Networking(mobiCom), 183-192.

[9] C. F. Chiasserini, I. Chlamtac, A. Nucci, "Energy efficient design of wireless ad hoc networks", in Proc. the 2nd IFIP networking. Vol. LNCS 2345, 367-386. 2002

[10] S. Kumar, A. Arora, T. H. Lai. "On the lifetime analysis of alwayson wirless sensor network applications", in Proc. the IEE International Conference on Mobile Ad-hoc and sensor systems (MASS), Washington, November 2005.

[11] S. Goel, T. Imielinski, "Prediction-based Monitoring in Sensor Networks: Taking Lessons from MPEG". Jal of ACM Computer Communication Review, Vol. 31, 2001

[12] L. Liu, P. S. Yu, "ASAP: An Adaptive Sampling Approach to Data Collection in Sensor Networks". IEEE Transactions on Parallel and Distributed Systems, 2007.

[13] S. Goel, A. Passarella, T. Imielinski, "Using buddies to live longer in boring world", in Proc. the Fourth Annual IEEE International Conference on Pervasive Computing and Communications Workshops, 2004.

[14] R. Willett, A. Martin, R. Nowak, "Backcasting: adaptive sampling for sensor networks", in Proc. the 3rd international symposium on Information processing in sensor networks (IPSN'04), 2004.

[15] A Djafari Marbini, L. E. Sacks, "Adaptive Sampling Mechanisms in Sensor Networks", in Proc. LCS, 2003.

[16] A. Jain, E. Y. Chang, "Adaptive sampling for sensor networks", in Proc the 1st international workshop on Data management for sensor networks: in conjunction with VLDB, 2004.

[17] N. Kimura, S. Latifi, "A Survey on Data Compression in Wireless Sensor Networks", in Proc. International Conference on Information Technology: Coding and Computing (ITCC'05), 2005, pp.8-13.

[18] W. R. Heinzelman, A. Ch , H. Balakrishnan, "Energy-efficient communication protocol for wireless microsensor networks", in Proc. the 33rd Hawaii International Conference on System Sciences (HICSS'00), 2000.

[19] W. B. Heinzelman, "An application-specific protocol architecture for wireless networks“. IEEE TRANSACTIONS ON WIRELESS COMMUNICATIONS, Vol. 1, No. 4, October 2002.

[20] K. Akkaya, M. Younis, "A survey on routing protocols for wireless sensor networks“. Ad Hoc Networks Volume: 3, Issue: 3, Pages: 325 349, 2005. 
[21] W. Zhang, G. Cao, "Optimizing tree reconfiguration for mobile target tracking in sensor networks", in Proc. INFOCOM 2004, 2004.

[22] W. Zhang, G. Cao, "DCTC: dynamic convoy tree-based collaboration for target tracking in sensor networks". IEEE Transactions on Wireless Communications, 2004.

[23] A. Goel, D. Estrin, "Simultaneous optimization for concave costs: single sink aggregation or single source buy-at-bulk", in Proc. SODA, 2003.

[24] R. C. Baltasar, R. Cristescu, B. B. Lozano, M. V, "On Network Correlated Data Gathering", in Proc. INFOCOM 2004, 2004.

[25] Yujie Zhu, Sundaresan, K., Sivakumar, R, "Practical limits on achievable energy improvements and useable delay tolerance in correlation aware data gathering in wireless sensor networks", in Proc. IEEE SECON 2005, 2005.

[26] K. W. Fan, S. Liu, P. Sinha, "On the potential of Structure-free Data Aggregation in Sensor Networks", in Proc. INFOCOM 2006, April 2006.

[27] K. W. Fan, S. Liu, P. Sinha, "Scalable data aggregation for dynamic events in sensor networks", in Proc. the 4th international conference on Embedded networked sensor systems (SenSys'06), 2006.

[28] S. Mahfoudh, P. Minet, "An energy efficient routing based on OLSR in wireless ad hoc and sensor networks", in Proc. PAEWN08, Okinawa, Japan, March 2008.

[29] http://tools.ietf.org/html/rfc6206

[30] G. Pei, M. Gerla, T.-W. Chen, "Fisheye state routing: a routing scheme for ad hoc wireless networks", in Proc. IEEE ICCO0, New Oreleans, LA June 2000.

[31] van Der Schaar, M. Sai Shankar N, "Cross-layer wireless multimedia transmission: challenges, principles, and new paradigms". IEEE Wireless Communications, vol. 12, 2005, pp. 50-58.

[32] M. Garey, D. Johnson, Computers and intractability: a guide to the theory of NP-completeness, Freeman, San Francisco, 1979.

[33] J. Wu, H. Li, "On calculating connected dominating set for efficient routing in ad hoc wireless networks, in Proc. the $3 r d$ international workshop on Discrete algorithms and methods for mobile computing and communications DIALM '99, New York, August 1999.

[34] F. Dai, J. Wu, "An extended localized algorithm for connected dominating set formation in ad hoc wireless networks“. IEEE Trans. on Parralle and distributed systems, vol. 15(10), 2004.

[35] F. Ingelrest, D. Simplot-Ryl, I. Stojmenovic, "Smaller Connected Dominating Sets in Ad Hoc and Sensor Networks based on Coverage by Two-Hop Neighbors“, in Proc. 2nd International Conference on Communication System Software and Middleware, Bangalore, India, 2007.

[36] K. Alzoubi, P.J.Wan, O. Fieder, 'Distributed heuristics for connected dominating sets in wireless ad hoc networks“. Jal of Communications and Networks, vol. 4(1), March 2002.

[37] B. Han, H.H. Fu, L. Li, W. Jia, "Efficient construction of connected dominating set in wireless ad hoc networks“, in Proc. IEEE MASS 2004 Fort Lauderdale, Florida, October 2004.

[38] E.-O. Blass, J. Horneber, M. Zitterbart, "Analyzing Data Prediction in Wireless Sensor Networks", in Proc. IEEE Vehicular Technology Conference, VTC Spring 2008, 2008.

[39] K. Zeng, W. Lou, J. Yang, D. R. Brown III, ”On Geographic Collaborative Forwarding in Wireless Ad Hoc and Sensor Networks", in Proc. International Conference on Wireless Algorithms, Systems and Applications (WASA), 2007.

[40] Jun Luo; Hubaux,J.-P., "Joint mobility and routing for lifetime elongation in wireless sensor networks“, in Proc. 24th Annual Joint Conference of the IEEE Computer and Communications Societies (INFOCOM 2005). 2005.

[41] M. Bhardwaj and A.P. Chandrakasan, "Bounding the Lifetime of Sensor Networks Via Optimal Role Assignments“, in Proc. the 21st IEEE INFOCOM, 2002

[42] M. Cagalj, J.-P. Hubaux, and C. Enz, "Minimum-energy Broadcast in All-wireless Networks: NP-completeness and Distribution Issues", in Proc. the 8th ACM MobiCom, 2002.

[43] I. Papadimitriou, L. Georgiadis, "Energy-aware Routing to Maximize Lifetime in Wireless Sensor Networks with Mobile Sink“. Journal of Communications Software and Systems, vol. 2 (2), pp. 141-151, 2006.

[44] L. Pelusi, A. Passarella, M. Conti, "Opportunistic Networking: Data Forwarding in Disconnected Mobile Ad Hoc Networks“. IEEE Communications Magazine, vol.44, no. 11, pp.134-141, Nov.2006.

[45] R.C. Shah, S. Roy, S. Jain, W. Brunette, "Data MULEs: modeling a three-tier architecture for sparse sensor networks", in Proc. IEEE International Workshop on Sensor Network Protocols and Applications (SNPA 2003), May 11, 2003, pp. 3041.
[46] C. H. Ou, K. F. Ssu, "Routing with mobile relays in opportunistic sensor networks", in Proc. the 18th Annual IEEE International Symposium on personal, indoor and Mobile radio Communicatioons (PIRMC'07), 2007.

[47] S. Meguerdichian, M. Potkonjak, "Low power 0/1 coverage and scheduling techniques in sensor networks", UCLA Technical Reports 030001 , January 2003.

[48] K. Chakrabarty et al., "Grid coverage for surveillance and target location in distributed sensor networks". IEEE Transactions on Computers, vol 51 (12), 2002, pp. 1448-1453.

[49] Y. Xu, J.Heidemann, D.Estrin, "Adaptive energy-conserving routing for multihop ad hoc Networks", Research Report 527, USC/Information Sciences Institute, October 2000.

[50] A. Cerpa, D. Estrin, "ASCENT: Adaptive Self-configuring Sensor Networks Topologies", in Proc. the 21st Annual Joint Conference of the IEEE Computer and Communications Societies, New York, USA, June 2002.

[51] B. Chen, K. Jamieson, H. Balakrishnan, R. Morris, "Span: an energy efficient coordination algorithm for topology maintenance in ad hoc wireless networks“. ACM Wireless Networks, vol. 8 (5), 2002

[52] X. Wang, G. Xing, Y. Zhang, C. Lu, R. Pless, C. Gill, ”Integrated coverage and connectivity configuration in wireless sensor networks", in Proc. ACM SenSys03, Los Angeles, California, USA, November 2003.

[53] M. Cardei, D. Du, "Improving wirelessn sensor network lifetime through power aware organization“. ACM Journal of Wirless Networks, May 2005.

[54] M. Cardei, M. Thai, Y. Li, W. Wu, "Energy-efficient target coverage in wireless sensor networks", in Proc.IEEE INFOCOM 2005, Miami, Florida, March 2005

[55] V. Rajendran, K. Obraczka, and J. j. Garicia-Luna-Aceves, "EnergyEfficient, Colision-free Medium Access Control for Wireless Sensor Networks“, in Proc. ACM SenSys'03, Los-Angeles, CA, Nov.2003, pp. 181-192.

[56] V. Rajendran, "Energy-efficient, application aware medium access for sensor networks", in Proc. 2nd IEEE Conf. on Mobile Ad-hoc and Sensor Systems (MASS'05), 2005.

[57] W.L. Lee, A. Datta, R. Cardell-Oliver, "FlexiTP: A Flexible-ScheduleBased TDMA Protocol for Fault-Tolerant and Energy-Efficient Wireless Sensor Networks". IEEE Transactions on Parallel and Distributed Systems, Vol. 19(6), pp. 851-864, June 2008

[58] S. Gobriel, D. Mousse, R. Cleric, "TDMA-ASAP: sensor network TDMA scheduling with adaptive slot stealing and parallelism", in Proc.ICDCS 2009, Montreal, Canada, June 2009

[59] W. Ye, J. Heidemann, D. Estrin, "Medium access control with coordinated adaptive sleeping for wireless sensor networks". IEEE/ACM Transactions on Networking, vol. 12(3), pp. 493-506, June 2004.

[60] T. van Dam and K. Langendoen. "An adaptive energy efficient MAC protocol for wireless sensor networks", in Proc. 1st International Conference on Embedded Networked Sensor Systems, pages 171180. ACM, November 2003.

[61] G. Lu, B. Krishnamachari, C.S. Raghavendra, "An adaptive energy efficient and low-latency Mac for data gathering in wireless sensor networks", in Proc. 18th International Parallel and Distributed Processing Symposium, New Mexico, USA, April 2004, pp. 224, 26-30.

[62] J. Polastre, J. Hill, D. Culler, "Versatile low power media access for sensor networks", in Proc. Second ACM Conference on Embedded Networked Sensor Systems (SenSys), Maryland, November 2004.

[63] I. Rhee, A. Warrier, M Aia, J. Min, "Z-MAC: a hybrid MAC for wireless sensor networks", in Proc. ACM SenSys 2005, S Diego, USA, November 2005

[64] S. Mahfoudh, G. Chalhoub, P. Minet, M. Misson, I. Amdouni, "Node Coloring and Color Conflict Detection in Wireless Sensor Networks". Future Internet, vol. 2(4), pp. 469-504, 2010.

[65] Miguel A. Labrador, Pedro M. Wightman, Topology Control in Wireles Sensor Networks with a companion simulation tool for teaching and research, Springer Science and Business Medi, 2009

[66] F. Ingelrest, D. Simplot-Ryl, I. Stojmenovic, "Optimal Transmission Radius for Energy Efficient Broadcasting Protocols in Ad Hoc Networks". IEEE Transactions on Parallel and Distributed Systems, June 2006.

[67] R. Zhang, M.A, Labrador, "Energy-aware topology control in heterogeneous wireless multihop networks", in Proc. 2nd IEEE International Symposium on Wireless Pervasive Computing, Puerto Rico, 2007. 